\title{
EN TORNO AL CONCEPTO DE SUBSTANCIA EN LOCKE
}

En este artículo examinaremos la concepción que Locke tiene de la substancia en tanto que ésta se refiere a objetos materiales. El objeto de la investigación será descubrir, por medio de comparaciones históricas, cómo y por qué Locke entendió incorrectamente el concepto de substancia. Más concretamente, veremos que Locke entendió incorrectamente el concepto de substancia por dos razones principales: a) tomó elementos de los conceptos de substancia de Aristóteles y de Descartes, sin darse cuenta que estos conceptos y las doctrinas metafísicas y epistemológicas en las que se inscriben, son incompatibles; y b) intentó, de manera incompleta, desarrollar una concepción empirista de la substancia. Ya que el concepto de substancia generalmente es introducido para resolver varios problemas, a saber, los de sujeto lógico, unidad, clasificación, individuación y unidad temporal (cfr. [12], pp. 3-6, aunque nuestra división de problemas es un poco distinta), examinaremos algunos de ellos por separado, ${ }^{1}$ centrándonos en las tesis de Locke.

Para comprender las razones que llevaron a Locke a postular un substrato incognoscible o substancia debemos comparar las distintas visiones del mundo de Aristóteles y Descartes, ya que éstas forman parte de su teoría filosófica, y la impulsan en direcciones opuestas. Para Aristóteles, y la Escolástica, el conocimiento era básicamente clasificatorio. Asociada a esta tesis epistemológica se encontraba la siguiente tesis metafísica: cualquier entidad que tiene existencia propia o independiente, esto es, que es una substancia, es un compuesto de materia y forma. La forma puede ser esencial o accidental. La esencia nos dice qué cosa es aquello que es, es decir, nos dice a qué clase o especie pertenece la cosa en cuestión. En contraposición con la substancia, caracterizada por la esencia, están los accidentes (tales como cantidades, cualidades, relaciones, etc.): los accidentes no tienen una existencia independiente ya que existen en, o inhieren en ("inhere" es la palabra técnica que Locke utilizó) la substancia como caracteristicas o modificaciones de ésta. Por ejem. plo, el doblez de un pedazo de papel es un accidente de ese pedazo de papel porque el doblez existe en, o es una modificación de, ese pedazo de papel. Así pues, el decir de cualquier substancia que tiene una existencia independiente es decir que la substancia no depende de la existencia de otra entidad de la cual sería una modificación, i.e., no requiere de otra entidad para existir

1 No examinaremos el problema del sujeto lógico, ya que las razones que llevaron a Locke a postular un substrato o substancia no fueron lingüisticas sino metafísicas y epistemologicas. 
como caracteristica de ésta, ${ }^{2}$ doctrina que, claramente, no niega la posibilidad de una interacción causal entre substancias. Ahora bien, la materia absolutamente indeterminada (o materia prima) subyace a la forma de una manera distinta a la que la substancia subyace a los accidentes ([10], p. 200), ya que la materia prima es indeterminada, no es una entidad con existencia propia, y por tanto no puede funcionar como un soporte de la forma; es decir, la forma no puede "existir en" la materia; más bien, la materia prima subyace a la forma en el sentido de que la forma determina y actualiza aquello que es indeterminado y potencial. Por otro lado, la substancia es un soporte para, o entidad en la que inhieren los accidentes, ya que la substancia es una entidad determinada, y con existencia propia, de la cual los accidentes son modificaciones. Ahora bien, la razón por la cual dijimos que la concepción aristotélica de la substancia estaba relacionada con su visión clasificatoria del conocimiento, radica en el hecho de que la forma substancial o esencia, que es un componente de cualquier substancia, es la razón metafísica por la cual las substancias pueden ser clasificadas, i.e., por la cual diversos individuos pueden pertenecer a la misma clase.

Para Descartes, por el contrario, el conocimiento, especialmente el tipo de conocimiento que ofrece la f́sica mecanicista, ${ }^{3}$ no consiste en la clasificación de diversas substancias sino en la descripción matematizada de las propiedades y relaciones entre substancias materiales que están en movimiento o descanso. Correspondiendo con esta posición epistemológica, en el plano ontológico Descartes sostuvo que el mundo material está constituido por substancias que se definen sólo en términos de propiedades matemáticas o cuantitativas (o "cualidades primarias"). Descartes expresó esta tesis afirmando que la esencia de la substancia material es la extensión o tridimensionalidad, de tal modo que las únicas propiedades de una substancia material son el tener un volumen de determinada magnitud, el tener una forma (geométrica), y el moverse o estar en descanso. De esta tesis se sigue que las substancias materiales pueden diferir en número pero no en tipo ([13], p. 129). En verdad, para Descartes las substancias materiales no pueden pertenecer a distinto tipo o clase de cosa, ya que por ser todas ellas esencialmente objetos extensos, sólo pueden diferir cuantitativamente. Por ejemplo, la distinción que ordinariamente hacemos entre un pájaro y una piedra no es para él una diferencia entre dos tipos de cosas, sino meramente entre dos pedazos de materia que tienen distinta cantidad de materia o de movimiento, o distinta forma geo métrica, es decir, es sólo una diferencia numérica entre dos pedazos de materia.

2 El término "existencia propia" o "existencia independiente" también significa existencia extramental. Por ejemplo, los números no son substancias para Aristóteles porque no tienen existencia extramental.

3 Por ffíca mecanicista entendemos aquella física que estudia a la naturaleza sólo como materia en movimiento. 
Aristóteles, en cambio, estaba interesado en tener una ontología en que los objetos difiriesen en tipo, o sea, perteneciesen a distintas clases, ya que dicha ontología correspondería a su visión clasificatoria del conocimiento. En dicha ontología las substancias son metafísicamente analizadas en una forma substancial, o esencia, la cual da cuenta de por qué las substancias son de un tipo u otro; una materia (designada) que explica cómo es posible que existan individuos numéricamente distintos del mismo tipo; y un conjunto de propiedades accidentales gracias a las cuales podemos distinguir substancias tanto cualitativamente, i.e., en términos de tipos, como numéricamente (por ejemplo, la risa o el lenguaje manifiestan un ser racional; el pelo negro, la gordura, etc., nos ayudan a distinguir a una persona de otra).

En Descartes encontramos un concepto de substancia radicalmente distinto al aristotélico. A Descartes lo que le interesaba era dar cuenta de la física mecanicista, la cual trata a los objetos f́́sicos no como diferenciables en términos de especies y géneros sino sólo como diferenciables numéricamente en tanto que ejemplifican distintas propiedades o relaciones matemáticas (tales como distintas magnitudes, formas geométricas o posiciones relativas a otros cuerpos). Correspondiendo a esta nueva visión del conocimiento -mecanicista - encontramos que Descartes no analiza metafísicamente a la substancia en materia y forma, y a la forma en esencia y accidente, sino que la analiza en términos de atributo (o esencia) y sujeto (o substancia). Este concepto de substancia no puede ser usado para indicar la existencia de distinciones de clase o tipo, sino sólo para hacer distinciones categoriales, es decir, distinciones entre la substancia material y la mental, las cuales tienen distinto atributo o esencia.

Veamos ahora las razones por las que se introduce el concepto de substancia en varios sistemas filosóficos. Una de las razones que Descartes dio en favor de la distinción sujeto-atributo es que el atributo o esencia debe serlo de alguna cosa; y, más aún, para ser existente, el atributo debe inherir en alguna cosa existente o substancia (sujeto) ([11], pp. I, LII). En Locke encontramos un argumento similar para introducir la noción de substancia:

porque no podemos concebir cómo ellas [las ideas simples de cualidades sensibles] pueden subsistir solas, ni una en la otra, suponemos que ellas existen en y son soportadas por un sujeto común; a este [sujeto o] soporte lo denotamos por medio del nombre de "substancia" ([6], $\mathrm{n}, \mathrm{xxm}, 4)$; y la mente percibe que las ideas de estas cualidades y acciones o poderes son, por sí mismas, inconsistentes con la existencia... Dado que la mente percibe su conexión necesaria con la inherencia o con el ser soportadas, la cual es una idea relativa, superpuesta al color rojo en una cereza o al pensamiento en un hombre, la mente fabrica la idea correlativa de un soporte, [lo cual es posible] porque nunca he negado que la mente pueda fabricar para ella misma ideas de relación, sino que he 
mostrado todo lo contrario en mis capitulos acerca de las relaciones (primera carta a Stilingfleet, citada en [9], p. 77; el subrayado es mío).

Este argumento de Locke presenta las siguientes fallas: 1) No resulta claro por qué existe una conexión necesaria entre las ideas de cualidades y la idea de substancia (o substrato), ya que no es claro por qué las cualidades no pueden existir por sí mismas, como los fenomenalistas sostienen. Nótese que esta obscuridad no está presente en Aristóteles, dado que lo que él sostiene es que los accidentes necesitan un soporte, una substancia compuesta de forma y materia, porque ellos son solamente modificaciones o características y, por ende, no pueden tener existencia propia. 2) La substancia o soporte se convierte en una cosa, que tiene existencia independiente, y la mente, al "superponer" esta cosa a otras cosas, tales como cualidades, entiende cómo las cualidades pueden existir. Esta línea de argumentación es criticable porque hace del substrato algo incognoscible ([6], II, xxm, 2). Finalmente, 3) es difícil entender cómo un empirista, o un racionalista, puede sostener que la mente forma una relación entre cosas que son empíricas o racionalmente incognoscibles - tal como el substrato.

El concepto de substancia también es introducido para dar cuenta del problema tradicional de la unidad de las propiedades en una cosa. Por ejemplo, para Aristóteles varias propiedades accidentales son propiedades de una misma cosa porque ellas inhieren en una substancia. Debemos notar que en el caso de las propiedades esenciales el problema de la unidad no surge porque cada cosa tiene sólo una esencia. Para ilustrar esto tomemos como ejemplo la tesis aristotélica de que la esencia del hombre es ser un animal racional; vemos aquí que no existe el problema de cómo la animalidad y la racionalidad están integradas en una sola cosa porque animalidad y racionalidad no son dos propiedades (esenciales) diferentes -en verdad, ser racional es sólo una forma particular de ser animal. Por el contrario, en el caso de las propiedades accidentales no hay una conexión necesaria de ellas entre sí mismas o de ellas con la esencia, por lo cual surge la pregunta de por qué existen juntas formando un objeto unitario. La respuesta aristotélica es que ellas inhieren en, o son modificaciones de, la misma substancia.

En Descartes, el concepto de substancia no es usado para resolver el problema de la unidad dado que para él no existe tal problema ([14], p. 406). Descartes sostiene que las substancias materiales sólo tienen cualidades primarias, i.e., modos del atributo o esencia extensión, y no secundarias, por lo cual él no postula accidentes que requieran ser unificados. La razón por la cual las cualidades secundarias (tales como color, calor, suavidad, etc.) no pueden ser accidentes de la substancia material es que ellas no son propiedades de la substancia material por no ser modos del atributo extensión o tridimensionalidad, i.e., por no ser cuantitativas. Tampoco las cualidades primarias son 
accidentes para Descartes, por la siguiente razón. El atributo o esencia de la substancia material es la extensión, esto es, el cuerpo es extensión. Los modos son la forma particular en la cual la extensión se presenta, esto es, los modos son la forma concreta en la cual el cuerpo o extensión existe - por ejemplo, un cuerpo particular es una determinada magnitud, es una figura determinada, etc. Dado que para Descartes los modos de un atributo son la forma particular en la cual el atributo se presenta, los modos, o cualidades primarias en el caso de la substancia material, no son accidentes por no ser modificaciones o propiedades de los cuerpos, sino que son solamente formas particulares de ser, o existir como, cuerpo.

Locke también utiliza el argumento de la unidad de las propiedades para introducir la substancia o substrato:

uno no tiene otra idea de cualquier substancia, v.g., de oro, caballo, hierro, hombre, vitriolo, pan, sino la que tiene de estas cualidades sensibles, que uno supone que inhieren; con la suposición de un substrato que soporta, por asf decirlo, a estas cualidades o ideas simples, que uno observa que existen unidas ([6], II, XxiII, 6; cfr. también $\mathrm{n}, \mathrm{xxII}, 1$, 14 y 37).

Una de las razones por las cuales Locke utiliza el argumento de la unidad es la misma razón metafísica que Aristóteles dio: dado que los accidentes no son parte esencial de la substancia, necesitamos una razón que de cuenta de su estar unidos formando un solo objeto. Locke sugiere que es este argumento metafísico el que tiene en mente cuando identifica las cualidades con los accidentes ([6], II, xxuI, 2 y 15). Sin embargo, el uso de Locke del argumento aristotélico es incorrecto porque, al adoptar la tesis mecanicista cartesiana acerca de los objetos materiales, se queda sin accidentes que requieran ser unificados. Como hemos visto, de acuerdo con la tesis mecanicista cartesiana, las cualidades secundarias no pueden ser accidentes de los objetos materiales dado que no son propiedades de ellos. Con respecto a las cualidades primarias podría pensarse que para Locke son accidentes porque él sostiene que la esencia o característica fundamental del cuerpo no es la extensión sino la solidez ([6]], II, XIII, 11 y 12), y sostiene que la magnitud, la figura, etc., son afecciones de los cuerpos ([6], II, XII, 3, nota 1 ; y 25). Sin embargo, dado que la solidez ${ }^{4}$ no es una propiedad (esencial) tal que las substancias pudiesen ser definidas en términos de ella y así ser conocidas, Locke se ve forzado a sostener que las cualidades primarias son las cualidades por medio de las cuales la substancia es conocida y que, por tanto, son las cualidades que constitu-

4 Por solidez Locke entiende aquello que hace que un cuerpo llene un lugar en el espacio y que presente resistencia a ser movido ([6], $\mathrm{I}, \mathrm{xm}, 11$ y 12); en otras palabras, Locke alude al concepto newtoniano de masa con su concepto de solidez. 
yen la esencia de los cuerpos. Así pues, las cualidades primarias son para Locke propiedades esenciales y, por ello, no pueden ser accidentes.

Es de suma importancia notar que el contexto particular en el que Locke usa los argumentos de inherencia (las cualidades necesitan un soporte o substrato en el cual existir) y de unidad (las cualidades necesitan un substrato que las unifique), lo lleva a entender al substrato, o substancia pura en general, como un "no sé qué" ("I know not what"). Recordemos que Aristóteles tiene una distinción entre propiedades esenciales y accidentales, según la cual las propiedades que requieren de un soporte para existir, o de un principio de unidad, son las accidentales, mientras que la substancia, determinada por la esencia, es aquella cosa existente que proporciona el soporte para, y unifica a, los accidentes. Ahora bien, la razón por la cual los argumentos de inherencia y unidad que Locke utiliza lo conducen a sostener que la substancia es incognoscible por ser una cosa carente de cualidades es que, dado que él no tiene una distinción entre propiedades accidentales y esenciales, son todas las propiedades o cualidades las que requieren ser soportadas y unificadas $y$, consecuentemente, no hay propiedades o cualidades restantes que puedan caracterizar al substrato, o substancia. Conviene destacar aquí que para Locke el substrato es una cosa existente, un ente, aun cuando carece de cualidades, o determinaciones, porque si el substrato ha de tener la función de soportar o conferir existencia a las cualidades, y la función de conferir unidad por ser una cosa en la cual ellas inhieren, tiene que ser alguna cosa existente. Y, claramente, si este substrato es una cosa existente carente de cualidades o determinaciones, se convierte en un "no sé qué": "la substancia se supone siempre como algo aparte de la extensión, figura, solidez, movimiento, pensamiento o cualquier otra idea observable, a pesar de que no sabemos qué es" ([6], II, xxuII, 3).

Alguien podría sostener que el que el substrato o substancia, según lo entiende Locke, sea incognoscible, no presenta problema alguno. Por ejemplo, en el sistema metafísico aristotélico la materia prima es incognoscible, sin que esto constituya una falla en tal sistema, ya que es posible hablar de la existencia de algo sin que conozcamos (o podamos conocer) ninguna de sus características. Podemos replicar a una persona que sostuviese esto lo siguiente. En el caso del sistema metafísico aristotélico no se propone que la materia prima sea un ente o una entidad con existencia propia. En verdad la materia prima nunca existe por sí sola, sino siempre determinada por una forma. Es decir, lo que existe para Aristóteles son substancias o individuos concretos que podemos analizar metafísicamente (i.e., sólo de modo intelectivo, sin que este análisis corresponda con entidades $\mathrm{u}$ objetos que existen como entidades distintas y que componen una substancia), en materia prima y forma. Ahora bien, para Aristóteles lo que podemos conocer son sólo substancias particulares (concretamente, aquello que ellas tienen en común con otras substancias 
particulares de su misma clase, es decir, su esencia), ya que éstas son lo único que tiene existencia independiente. Para Aristóteles no podemos conocer la materia prima misma, o la forma misma, no porque éstas por alguna razón u otra nos resulten incognoscibles, sino porque, en principio, no están sujetas a ser conocidas. En verdad, tanto la materia prima como la forma no tienen existencia propia, no son objetos en la ontologia aristotélica, sino que son meramente aspectos de objetos que un análisis metafísico revela, y por ende no son algo que cae dentro del campo de lo cognoscible (en principio o de hecho). En cambio, para Locke el substrato o substancia no tiene las dos características que acabamos de mencionar respecto de la materia prima aristotélica. $\mathrm{O}$ sea, el substrato de Locke tiene las siguientes dos caracteristicas: $a$ ) es una entidad existente por sí misma y $b$ ) pertenece al campo de lo cognoscible (en principio). Estas dos características del substrato hacen de él una entidad sospechosa, y más aún, inaceptable. Veamos.

El substrato como una cosa existente, carente de cualidades o determinaciones, es ontológicamente inaceptable por la siguiente razón. Locke sostiene que el substrato es un puro o mero ser: "la idea de la substancia en general es una idea compleja constituida de la idea general de entidad o ser, junto con la relación de ser sostén para los accidentes". (De una carta a Stilingfleet, citada en [1], p. 175.) Ahora bien, es difícil aceptar, como dice Berkeley, que pueda existir algo que sea indeterminado, esto es, algo que sea mera existencia y no existencia como hombre, o como pelota, etc. Y la razón por la cual es diffcil aceptar en una ontología una entidad tal como el substrato es que, por ejemplo, no tendríamos en principio criterio alguno de individuación, o de identidad temporal para tal entidad por carecer ésta de determinaciones. (Notemos que en el caso de Aristóteles esta objeción no se presenta porque él nunca sostuvo que la materia prima fuese una entidad, i.e., un objeto con existencia propia.)

La razón por la cual deberfamos tener algún criterio de individuación, o de identidad temporal, etc., es que estos criterios funcionan como principios de aceptabilidad de una entidad como tal dentro de cualquier teoria metafísica; y funcionan como principios de aceptabilidad porque la satisfacción de éstos sirve para especificar qué entidades sostenemos que existen en un esquema metafísico. Así por ejemplo, el que una entidad (o tipo de entidades) satisfaga algún criterio de individuación es de gran importancia porque esto nos permite especificar si la entidad que se dice existir es una o son varias; el que satisfaga algún criterio de identidad temporal nos informa que tal entidad es temporal y sujeta al cambio.

Por otra parte, la comprensión epistemológica de Locke del substrato es también inaceptable. Como sabemos, Locke es un empirista. ${ }^{5}$ Me parece que

5 Locke sostuvo que las dos fuentes del conocimiento son la observación de los objetos sensibles externos y la percepción de las operaciones internas de nuestra mente ([6], II, I, 2). 
esta posición empirista es la que llevó a Locke a comprender el substrato de manera inaceptable -inaceptable porque concibió al substrato como una entidad incognoscible en principio. En verdad, Locke parece suponer que el análisis de los objetos materiales en términos de substrato y cualidades es un análisis empírico (y no metafísico), es decir, que en realidad un objeto está compuesto por estos elementos, elementos que tienen existencia propia aun cuando, de hecho, siempre existan juntos. Me parece que esta interpretación es la correcta porque Locke llegó a identificar el substrato con la constitución atómica interna de los objetos materiales (cfr. más adelante). Ahora bien, una vez que se concibe este análisis como empírico, es legítimo preguntarse cómo y qué conocemos de cada uno de estos elementos que componen al objeto material. En el caso de las cualidades Locke no encuentra mucho problema: conocemos las cualidades vía los sentidos. En el caso del substrato, Locke evidentemente no encuentra ninguna vía de acceso a él, ya que no hay ninguna vía que nos permita conocer aquello que no tiene determinaciones o cualificaciones, de tal modo que el substrato se convierte en una entidad incognoscible en principio. En cambio, si Locke hubiese entendido este análisis como un análisis metafísico (que a veces sugiere que es, como cuando sostiene en su primera carta a Stilingfleet [ver pp. 82-3] que la mente percibe una conexión necesaria o racional entre substrato y cualidades fundadas, y por ello llega a saber que el substrato existe), se hubiera dado cuenta que la distinción entre substrato y cualidades es meramente racional y no corresponde a una "distinción real" entre elementos que componen un objeto. Y si se hubiese dado cuenta que esta distinción es meramente racional, habria notado que las partes distinguidas por el análisis no pertenecen al campo de lo cognoscible precisamente porque estas partes no son entidades. Asf pues, es la comprensión del substrato como entidad la que lleva a Locke a suponer que el substrato pertenece al campo de lo cognoscible, ya que por ser el substrato una entidad con existencia propia cabe preguntarse qué es, i.e., qué sabemos acerca de él y cómo lo sabemos; y por ser el substrato una entidad carente de determinaciones resulta ser un "no sé que".

Hemos sostenido que el substrato de Locke es epistemológicamente inaceptable por ser una entidad incognocible (en principio). Locke podría replicar a nuestra tesis sosteniendo que, como cree el sentido común, hay una brecha entre lo que conocemos y lo que existe (cfr. la primera carta a Stilingfleet en [6], nota 4, p. 394). Esta respuesta de Locke no es aceptable porque el substrato no es algo que hasta ahora no hemos conocido, sino que el substrato es algo incognocible en principio $\mathrm{y}$, por ende, no podemos tener razones para creer que existe. Ahora bien, es legítimo, por ejemplo, pensar que existen estrellas que no conocemos; pero esto es legítimo porque las estrellas son cognoscibles en principio, y por ende tenemos una idea, por general o vaga que ésta sea, acerca de cómo son dichas estrellas. En cambio, en el caso del substrato no 
es legitimo sostener que existe pero que no lo conocemos, porque la incognoscibilidad del substrato es en principio (por carecer de cualidades). Por ser el substrato incognoscible en principio, no podemos tener idea alguna de qué es $y$, por ende, no tiene sentido sostener que existe — ¿qué es lo que existe?

Para resumir, podemos decir que el error fundamental que Locke comete en su doctrina de la substancia es haber considerado que el substrato es una entidad $\mathbf{u}$ objeto con existencia propia. Esta consideración es errónea tanto porque no es correcto postular en una ontología entidades que carecen de determinación, i.e., cuya única característica es la de ser mera existencia, como porque, epistemológicamente, lleva a la aceptación de una entidad de la que no se puede saber nada en principio. La razón por la cual Locke concibe al substrato como una entidad $\mathbf{u}$ objeto con existencia propia es que él concibe el análisis de objetos materiales en términos de substrato y cualidades como un análisis empírico en términos de las entidades o elementos que componen un objeto complejo.

Sostuvimos arriba que la razón epistemológica que llevó a Locke a entender incorrectamente a la substancia o substrato como una entidad carente de cualidades fue su empirismo. Veamos cómo opera en otros casos el empirismo de Locke. En varios pasajes, él sugiere fuertemente que el problema de la unidad (que da lugar al análisis en términos de substrato y cualidades) no es un problema metafísico sino empírico, y que consiste en la pregunta: ¿cuál es la causa empírica de que las cualidades sensibles se encuentren unificadas de tal modo que forman una sola cosa? Locke nos da a entender que el substrato, que es una cosa existente determinada por ser la constitución interna atómica de los objetos materiales o substancias particulares, es la causa de la unidad de las cualidades:

el substrato donde ellas subsisten y del cual resultan, al cual por tanto llamamos substancia ([6], II, xxuI, 1; el segundo subrayado es mío); y todas las ideas que tenemos de tipos particulares distintos de substancia no son nada más que varias combinaciones de ideas simples, que coexisten en la causa, aunque no conocida, de su unión ([6] II, XxIII, 6; y II, xxm, 3, donde explica que la causa-substrato es la constitución interna atómica de las cosas).

Claramente, si se pudiese identificar el substrato con la constitución interna atómica de las substancias particulares, el substrato resultaría ontológicamente aceptable dado que sería una cosa existente determinada que sostiene y unifica cualidades observables en el sentido de que es la causa de que existan. El substrato también sería epistemológicamente aceptable dado que su incognoscibilidad seria meramente de hecho.

Sin embargo, Locke no puede identificar el substrato con la constitución 
atómica interna de los objetos materiales por la siguiente razón. El substrato que da cuenta de los problemas metafísicos de inherencia y unidad de cualidades carece de determinaciones y, por lo tanto, es incognoscible en principio; mientras que la constitución atómica interna está caracterizada por cualidades primarias ([6], II, xxIIr, 8-11) y su incognoscibilidad es sólo empírica, ya que se debe al hecho de que no tenemos ojos microscópicos ([6], II, xxuIr, 11 y 12).

Otro caso en el que Locke intenta, erróneamente, identificar el substrato entendido como ente sin determinaciones y el substrato como constitución atómica, es el caso de la clasificación. Recordemos que la noción de substancia y su anślisis en términos de materia y forma fue usada por Aristóteles para dar cuenta del problema de clasificación. Aristóteles sostuvo que los individuos particulares o substancias se agrupan en tipos o clases (naturales, i.e., no convencionales $o$ arbitrarias) por tener la misma forma - mientras que la materia explica por qué hay individuos numéricamente distintos del mismo tipo. Locke también usó su análisis de substancias particulares en términos de substrato o substancia y cualidades sensibles para dar cuenta del problema de la clasificación en tipos o clases: él sostuvo que el substrato o constitución atómica de los objetos materiales es aquel elemento en función del cual se debe clasificar a los objetos materiales ([6], III, vi, 2 y 3). Recuérdese que Locke sostiene que dado que tal constitución atómica, o esencia real, es de hecho desconocida, sólo nos queda la alternativa de clasificar substancias en términos de algún grupo de cualidades sensibles, o esencia nominal, que la constitución atómica causa ([6], III, vi, 3 y 9). Dada la respuesta de Locke podemos decir que él concibió el problema de la clasificación como un problema empírico - ¿qué elemento o parte de un objeto material es el que debemos usar para clasificarlo? - y no como un problema metafísico, como en el caso de Aristóteles — hay algún aspecto en las substancias particulares que justifique la creencia de que las clasificaciones que hacemos son naturales y no convencionales?

Por otra parte, Locke usó la noción de substrato, entendida como ente carente de cualidades, para dar cuenta de una clasificación categorial, i.e., de la clasificación de individuos en substancia material o substancia pensante. Así, por ejemplo, Locke sostiene:

Sin tener otra idea o noción de materia, sino como algo donde subsisten aquellas múltiples cualidades sensibles que afectan nuestros sentidos; al suponer una substancia donde el pensamiento, el conocer, el dudar, un poder de movimiento, y demás, subsisten, nosotros tenemos una noción tan clara de la substancia que es el espíritu, como la que tenemos de cuerpo; una se supone que es (sin saber qué es) el substrato para aquellas ideas simples que formamos de fuera; y la otra se supone (con igual ignorancia respecto de qué es) que es el substrato para aquellas operaciones que experimentamos dentro de nosotros mismos. ([6], II, XxIII, 5). 
La tesis que Locke sostiene en este pasaje es bastante peculiar: él dice que la substancia material es el substrato donde subsisten las cualidades sensibles, mientras que la substancia espiritual es el substrato donde subsiste el pensamiento, ¡como si la diferencia entre la substancia material y la pensante residiese en el substrato y no en las propiedades que inhieren en éll Sin embargo, supongamos que lo que Locke en realidad quiso sostener es la tesis más plausible de que la clasificación categorial en materia y espíritu es posible porque las cualidades sensibles inhieren siempre en un substrato distinto (numéricamente) al substrato en que inhieren los pensamientos; en otras palabras, materia y espíritu son dos entes distintos porque las propiedades materiales y las mentales inhieren siempre en distintos substratos.

Locke no puede usar el mismo sentido de substancia para hacer ambas clasificaciones. A una clasificación en tipos corresponde sólo el substrato como constitución atómica interna, ya que el substrato como carente de cualidades es el mismo en todos los individuos. A una clasificación categorial entre substancias mentales y materiales corresponde el substrato como algo carente de cualidades, y no el substrato como constitución atómica, ya que tal constitución lo es sólo de las substancias materiales.

No solamente Locke no puede usar el mismo sentido de substancia para dar cuenta de una clasificación en tipos y una clasificación categorial, sino que, y aun más importante, él no puede tener ambas especies de clasificación en su sistema. Veamos. Hemos dicho que el punto de vista mecanicista de Descartes acerca de la substancia material tiene por efecto el hacer que los objetos materiales difieran sólo en número pero no en tipo. Tiene este efecto porque se postula que las substancias materiales están caracterizadas sólo por propiedades (espaciales) cuantitativas, y los diversos modos de cualquiera de estas propiedades no difieren entre sí en términos de tipo. Por ejemplo, sería absurdo el sostener que un caballo y un hombre pertenecen a distintos tipos de substancias porque tienen distinta magnitud en largo, ancho y profundidad, o porque el número, tamaño y figura de los átomos o corpúsculos que los constituyen son distintos; y decimos que sería absurdo el sostener esto porque tales diferencias ocurren entre todos los objetos materiales (por ejemplo, dos objetos pueden diferir en su magnitud, figura y movimiento y, sin embargo, ambos ser hombres) y, consecuentemente, tales diferencias sólo pueden indicar una diferencia numérica (esto es, que hay dos o más objetos). Suponer que estas diferencias indican una distinción de tipos o clases de cosas sería identificar las distinciones de tipo con las distinciones numéricas, es decir, serf́a hacer vacuo el concepto de distinciones de tipos de cosas. Es importante destacar aquí que el objetivo de Descartes al sostener que el atributo de la substancia material es la extensión era, precisamente, eliminar la posibilidad de clasificaciones en términos de tipo, y con ello eliminar la explicación aristotélica vía formas substanciales. Su intención era la de substituir este tipo de 
explicación aristotélica por una nueva concepción de la explicación del mundo material y, más concretamente, por una nueva concepción de la legalidad o causalidad que rige al mundo. En verdad, para Descartes la ciencia o conocimiento del mundo material consiste en descubrir las leyes que rigen a todo el mundo material (leyes que él, perspicazmente, llamó "leyes de la naturaleza" ([11], pp. II, xxxviI)). Explicar, pues, consistiría en mostrar cómo un evento dado se sigue de estas leyes universales. Por supuesto, esta concepción de la ciencia está fundada sólo en la medida en que el mundo material sea uno, uniforme, y no dividido en clases naturales regidas por leyes particulares a cada clase, es decir, en la medida en que lo que existan sean substancias materiales que difieran numéricamente y no substancias que difieran esencialmente.

Ahora bien, como hemos visto, Locke adoptó la distinción categorial cartesiana entre la substancia material y la mental, y la comprensión mecanicista cartesiana de la substancia material, y dado que este punto de vista sólo nos permite hablar de substancias materiales que difieren en número pero no en tipo, la pretensión de Locke de tener una ontología de substancias materiales que difieren en tipo hace que su sistema sea inconsistente. Muy probablemente, esta inconsistencia se deba a que Locke intentó extender la concepción mecanicista cartesiana, que estaba propuesta en primer lugar para justificar a la física, a la química. Esta extensión resultó inválida porque, a la vez que Locke concebía a la química (o alquimia) como estudio de substancias heterogéneas (tales como alcohol, azufre, etc.), i.e., substancias que pertenecen a distintas clases, también retomó la concepción mecanicista cartesiana sin darse cuenta que ésta estaba especialmente diseñada para presentar al mundo material como homogéneo.

Aristóteles también usó la noción de substancia (como compuesto de materia y forma) para dar cuenta de los problemas metafísicos de individuación e identidad temporal. Locke, igualmente, trató estos problemas, pero su tendencia empirista (que notamos arriba) a reinterpretar problemas metafísicos como problemas físicos o empíricos ( $c f r$. en la p. 87 su tendencia a entender el análisis metafísico de la substancia como un análisis empírico) es aquí total, ya que él trató estos problemas como puramente empíricos, y consecuentemente no apeló a la noción de substancia para resolverlos. Ahora bien, el problema metafísico de la individuación surge para Aristóteles del hecho de que las formas substanciales y los accidentes son generales, esto es, son compartidos por varios individuos (y esto es lo que da cuenta de su pertinencia a distintas clases naturales) mientras que las cosas existentes son individuales o particulares. En la tradición aristotélica era la materia (designada) la que era el principio de individuación de las substancias que pertenecen a la misma clase, i.e., que tienen la misma forma substancial ([3], pp. 75-80). En cambio, en el caso de Locke encontramos que él no resuelve este problema metafísico, 
sino que más bien contesta la pregunta empírica "¿cuál es el criterio que, de hecho, usamos para distinguir entre dos objetos que pertenecen a la misma clase?" Su respuesta es que dicho criterio consiste en la ocupación de un lugar dado en cierto momento:

De lo que se ha dicho, es fácil descubrir aquello que tanto se busca, el principium individuationis; y éste, es claro, es la existencia misma; la cual determina a un ser de cualquier tipo a un tiempo y lugar determinados, incomunicables a dos seres del mismo tipo ([6], II, XxvII, 4).

Debemos notar que para Locke el hecho de que el criterio de individuación para objetos del mismo tipo sea la ocupación de un lugar determinado en un determinado momento se sigue del hecho de que tal ocupación es el criterio o condición necesaria y suficiente para la autoidentidad (o identidad estática): el "De lo que se ha dicho" de la última cita se refiere a parágrafos tales como el siguiente:

Cuando vemos que cualquier cosa en cualquier lugar en cualquier instante de tiempo, estamos seguros (sea lo que sea esa cosa) de que es esa misma cosa, y no otra que al mismo tiempo existe en otro lugar, tan semejante e indistinguible como pueda ser en todos los otros respectos: y en esto consiste la identidad ([6], II, xxvII, 1).

Locke usa la tesis de que el criterio para la autoidentidad o identidad estática es la existencia en un determinado lugar y tiempo, más el tipo de cosa al cual un objeto pertenece, para dar cuenta de la identidad temporal. Por ejemplo, él dice que dos partículas de materia (i.e., un átomo o una colección de átomos), o dos cuerpos (en tanto que son una masa de materia), que existen en distintos momentos, son una y la misma partícula de materia o uno y el mismo cuerpo si y sólo si hay una historia espacio-temporal continua que los une ([6], II, xxvIr, 2; [7], p. 141). Para ser más precisos, dado que en el caso de partículas de materia o de cuerpos, que se definen como trozos o agregados de materia, el único posible tipo de cambio que pueden tener y que nos permitiria hablar aún de la misma partícula de materia, es el cambio espacial (i.e., movimiento), el criterio para la identidad temporal es la historia espaciotemporal continua. En cambio, en el caso de las plantas, por ejemplo, que se definen como una organización de varias partes en una vida, es la vida vegetal espacio-temporalmente continua la que constituye criterio de identidad temporal ([6], II, Xxvir, 5).

Podemos decir que en el caso del problema de la identidad temporal Locke logró dejarlo de lado como un problema metafísico y tratarlo como un problema puramente empírico. Esto puede verse más claramente si notamos que, por ejemplo, los atomistas antiguos, que tomaron el problema de la 
identidad temporal como un problema metafísico, consideraron que podría ser resuelto si encontrásemos un elemento de la substancia que permaneciese siempre el mismo, sin ningún cambio (distinto al espacial), y otro elemento o elementos que cambian, y en virtud de los cuales podemos decir que la misma substancia cambió. Su solución consistió en sostener que lo que permanece siendo lo mismo son los átomos que constituyen la substancia, mientras que el cambio visible era sólo el reacomodo espacial de los átomos. Aunque Locke es un atomista, él no apeló a la inmutabilidad de los átomos para dar cuenta del problema de la identidad temporal, sugiriendo asi que no intentaba resolver este problema a un nivel metafísico.

Para terminar, haré algunos comentarios sobre la concepción de Locke acerca de la esencia real de las substancias materiales. Sostuvimos arriba que una de las características de Locke es que trató de reinterpretar problemas metafísicos como empíricos. Podemos sugerir que no lo logró porque su reinterpretación no fue lo suficientemente radical. Esto aparece muy claramente en su doctrina de la esencia real de las substancias materiales.

Por una parte él sostiene que la esencia real de la que habla es uno de los constituyentes empiricos de cualquier substancia material, dado que es la constitución interna atómica de cualquier substancia material; y consecuentemente, por esencia real él no se refiere a la esencia en sentido metafísico o forma substancial en sentido aristotélico ([15], pp. 101 y 130; [6], vol. II, nota 3 (de Fraser), p. 57). En verdad, él dice que:

Con respecto a las esencias reales de las substancias corpóreas (para mencionar sólo a éstas) hay, si no me equivoco, dos opiniones. La una es de aquéllos [i.e., los aristotélicos] que, al usar la palabra esencia para aquello de lo cual no saben nada [for they know not what], suponen un cierto número de estas esencias, de acuerdo con las cuales todas las cosas están hechas, y de las cuales cada una de ellas participa exactamente, y asi llega a ser de esta o aquella especie. La otra opinión, más racional, es de aquéllos que ven en todas las cosas naturales una constitución real, pero no conocida [unknown], de sus partes insensibles ([6], III, III, 17; $c f r$. también IIr, vx, 10 y 24, donde sostiene que las formas substanciales son ininteligibles $e$ inútiles).

En otras palabras, Locke no es un esencialista. El esencialismo es una posición metafísica que consiste en la tesis de que la naturaleza misma, independientemente de nuestra actividad clasificatoria, está organizada en tipos o clases (naturales) de cosas con límites precisos, organización que se debe a las formas substanciales o esencias. Locke critica la posición esencialista notando la existencia de monstruos (i.e., de entidades que no pertenecen a ninguna clase) en la naturaleza (cfr. [6], III, vi, 14-19 y 27).

Por otra parte, Locke sostiene que hay una conexión necesaria entre la 
esencia real y las cualidades observables de cualquier objeto material. Es decir, Locke sostiene que si conociésemos la esencia real de un objeto, sabríamos a priori cuales son sus cualidades observables (parte de las cuales están incluidas en la esencia nominal):

Yo no dudo que si pudiésemos descubrir la figura, tamaño, textura y movimiento de las diminutas partes que constituyen cualesquiera dos objetos, conoceríamos sin prueba alguna [without trial] varias de las operaciones que ejercen una sobre la otra; asf como conocemos las propiedades de un triángulo o de un cuadrado. Si conociésemos las afecciones mecánicas de las partículas del ruibarbo, la cicuta... seríamos capaces de decir por adelantado que el ruibarbo purga, la cicuta mata... Pero mientras no tengamos sentidos suficientemente agudos para descubrir las diminutas partículas de los cuerpos, y que nos den ideas de sus afecciones mecánicas, debemos contentarnos con ser ignorantes respecto de sus propiedades y sus modos de operación ([6], IV, III, 25; cfr. también VI, vr, 11, donde sostiene que podriamos conocer independientemente de cualquier observación o experimento, i.e., a priori, las cualidades observables de los objetos materiales, si conociésemos sus esencias reales).

Ahora bien, podemos observar que Locke ofrece por una parte un análisis empírico de la noción de esencia, pero por otra parte considera que su análisis tiene el mismo poder que la concepción aristotélica de la esencia, es decir, considera que la conexión entre la esencia real y las cualidades observables es necesaria y que, por ende, nosotros podemos conocer esta conexión de manera puramente racional, o a priori, o deductiva. Sin embargo, en una tradición esencialista o aristotélica, un conjunto de propiedades está necesariamente conectado con la esencia de una cosa no porque la esencia las cause, sino porque hay una conexión metafísica entre ellas: la esencia (metafísica) es lo que la cosa es, mientras que las propiedades $u$ operaciones se siguen de (es este "seguirse de" lo que el esencialista tiene que demostrar en cada caso particular), o manifiestan, el ser particular de la cosa ([8], p. 44; [15], p. 90). Así, por ejemplo, el que una planta se nutra se sigue de, o manifiesta, el alma vegetativa que la anima.

Ahora bien, dado que la esencia real de Locke no es una esencia en sentido metafísico, no puede estar conectada necesariamente con las propiedades observables como en el caso aristotélico. Por otro lado, dado que Locke es un empirista, y que su esencia real tiene un carácter físico, es muy difícil que podamos explicar inteligiblemente cómo, de la esencia real, podemos inferir a priori con cuál conjunto de cualidades observables está necesariamente conectada, por las siguientes razones. En primer lugar, el empirismo radical sostiene que no hay conexiones necesarias entre eventos $u$ objetos físicos porque tales conexiones no son observables. En segundo lugar, Locke no era un 
empirista radical, dado que él sostuvo que la mente es capaz de adquirir por sí misma conocimiento de conexiones necesarias - por ejemplo, sostuvo que la mente puede conocer por sí misma que las cualidades requieren necesariamente de un soporte o substrato, o que hay una conexión necesaria entre causa y efecto ( $c f r$. su primera carta a Stiligfleet citada en [6], vol. I, nota 1, p. 434). Pero aun si concedemos que Locke no era un empirista radical, para siquiera ser empirista él tendría al menos que sostener que las conexiones necesarias empíricas se establecen o verifican a través de la experiencia, en cuyo caso no puede sostener que, si conociésemos la esencia real de un individuo, podríamos saber a priori qué cualidades observables tiene el individuo en cuestión, sobre la base de que son causadas por la esencia real. En tercer lugar, aun si Locke hubiese sostenido una epistemología racionalista de algún tipo, debería haber explicado a nivel metafísico cómo son posibles las conexiones empíricas necesarias (por ejemplo, sosteniendo que son relaciones de identidad), cosa que no hizo. Así pues, podemos concluir que Locke da una interpretación empírica a la noción tradicional de esencia (a saber, la entiende como la constitución interna atómica de los objetos materiales); pero al sostener que hay una conexión necesaria y cognoscible (en principio) de manera a priori entre la esencia así comprendida y las cualidades sensibles, cae en una posición inconsistente. En cambio, si hubiese asociado su noción de esencia con una epistemología empirista (según la cual las conexiones son contingentes y sólo se pueden establecer vía un estudio empírico), su posición sería consistente.

Para concluir intentaremos descubrir cuál era el proyecto de Locke, a partir de lo visto anteriormente, y cuáles sus fallas principales.

Locke, al igual que la mayoría de sus contemporáneos, aceptaba como verdadera la física mecanicista (en especial, en la versión que Newton le dio). Por ello, tomó de Descartes dos tesis que le parecieron fundamentales en tanto que justificaban la física mecanicista, a saber, la tesis de la distinción real entre la substancia material y la pensante, y la tesis de que la substancia material sólo tiene cualidades primarias. Por otra parte, Locke, que era un médico interesado en la alquimia, intentó extender los conceptos básicos de la física mecanicista a la alquimia, aunque conservando la idea de que la alquimia era el estudio de diversas substancias agrupables en clases. Finalmente, Locke consideraba que el único modo como el conocimiento puede desarrollarse es sobre una base empírica. Estas tres ideas fundamentales de Locke, tal y como él las desarrolló, dieron lugar a una filosofía inconsistente, como puede notarse claramente en el concepto de substancia material de Locke.

Los errores principales en la doctrina de Locke acerca de la substancia material son los siguientes:

a) El haber considerado que el substrato o substancia es un ente con existen- 
cia propia que, por carecer de determinaciones, resulta incognoscible en principio.

b) El haber pensado que los conceptos de substancia de Aristóteles y de Descartes son compatibles, mientras que en verdad no son compatibles, ya que el primero sirve para hacer clasificaciones en tipos y el segundo para realizar clasificaciones categoriales. Estos conceptos están asociados a su vez con epistemologías incompatibles: para Aristóteles el conocimiento consiste básicamente en la clasificación, y para Descartes consiste en el descubrimiento de leyes universales. Locke intentó erróneamente integrar estas dos epistemologias, dado su intento de extender el modelo mecanicista de la física a la quimica.

c) Otro error de Locke consistió en haber sostenido que la esencia podía identificarse con un elemento material constitutivo de las substancias materiales (a saber, la constitución atómica) y que, a la vez, la relación entre esencia y accidentes es necesaria y puede conocerse a priori.

d) Los errores $a$ y $c$ se deben en gran medida a la epistemología empirista incompleta de Locke.

Euja Nathan

UNIVERSIDAD NACIONAL

Autónoma de Mexico

\section{BIBLIOGRAFIA}

1. Aaron, Richard I. John Locke. Second edition. Oxford: at the Clarendon Press. 1955.

2. Basson, A. H. "The Problem of Substance", in P.A.S., vol. XLIX, 1948-1949.

3. Bobik, Joseph. Aquinas on Being and Essence. A Translation and Interpretation. Indiana: University of Notre Dame Press. 1965.

4. Buchdahl, Gerd. Metaphysics and the Philosophy of Science. The Classical Origins: Descartes to Kant. Cambridge: the M.I.T. Press. 1969.

5. Gibson, James. Locke's Theory of Knowledge and its Historical Relations. Cambridge: at the University Press. 1931.

6. Locke, John. An Essay Concerning Human Understanding. Collated and annotated by A. C. Fraser. Vols. I and II. New York: Dover Publications, Inc. 1959.

7. Mackie, J. L. Problems from Locke. Oxford: Clarendon Press. 1976.

8. McCormick, John F. Scholastic Metaphysics. Part 1: Being, Its Division and Causes. Chicago: Loyola University Press. 1940.

9. O'Connor, D. J. John Locke. New York: Dover Publications, Inc. 1967.

10. Owens, Joseph. The Doctrine of Being in the Aristotelian Metaphysics. Toronto: Pontifical Institute of Medieval Studies. 1957. 
11. The Philosophical Works of Descartes. Rendered into English by Elizabeth S. Haldane and G. R. T. Ross. Vol. I. Cambridge: at the University Press. 1968.

12. Quinton, Anthony. The Nature of Things. London and Boston: Routledge and Kegan Paul. 1973.

13. Taylor, A. E. Elements of Metaphysics. London: Metheun and Co., Ltd. 1952.

14. Windelband, W. A History of Philosophy. New York: Harper. 1958.

15. Woolhouse, R. S. Locke's Philosophy of Science and Knowledge. A Consideration of some Aspects of An Essay Concerning Human Understanding. New York: Barnes and Noble. 197I. 